ORIGINAL ARTICLE

\title{
Success with hydrostatic reduction of intussusception in relation to duration of symptoms
}

\author{
E D van den Ende, J H Allema, F W J Hazebroek, P J Breslau
}

Background: It is widely believed that hydrostatic reduction of intussusception is less successful in children with prolonged symptoms prior to presentation.

Aim: To prospectively evaluate success in relation to duration of symptoms.

Methods: Prospective study in which children, regardless of symptom duration, underwent an attempt at hydrostatic reduction.

Results: Of 113 children presenting with intussusception, 16 had peritonitis and required immediate laparotomy. A hydrostatic reduction was attempted in 97 and was successful in $77(79 \%)$. There were 26 successful reductions with symptoms $<12$ hours $(81 \%), 30$ with symptoms for $12-24$ hours $(81 \%)$, and 21 with symptoms $>24$ hours $(75 \%)$.

Accepted 19 May 2005

Published Online First

21 June 2005

Conclusion: The success rate with hydrostatic reduction was not significantly influenced by symptom duration.

ntussusception is the most common abdominal emergency in early childhood, particularly in children younger than 2 years of age. ${ }^{1}$

It has been reported that successful hydrostatic reduction may be less likely in patients with symptoms for more than 48 hours, and consequently patients with prolonged symptoms are nowadays likely to undergo operative reduction as the first line treatment. ${ }^{1-3}$ Since there is little evidence to support this policy we undertook a prospective study to examine our success with hydrostatic reduction in relation to duration of symptoms.

\section{METHODS}

All children presenting to the Sophia Children's Hospital, Rotterdam or the Juliana Children's Hospital, The Hague, with ultrasound proven intussusception from January 1998 to December 2002 were included. A hydrostatic reduction was performed unless there was clinical or radiological evidence of peritonitis or perforation. Patient details, including nature and duration of symptoms, physical findings, laboratory results, and the findings on abdominal $x$ ray and ultrasound were prospectively recorded.

Hydrostatic reduction was performed using a standard protocol in which a $40 \mathrm{ml}$ balloon catheter was positioned in the rectum, and a reservoir containing water soluble contrast medium was positioned $100 \mathrm{~cm}$ above the patient and contrast then instilled into the colon. If reduction did not occur the contrast reservoir height was increased to $120 \mathrm{~cm}$. If this was unsuccessful the patient underwent a laparotomy.

Ethical approval for the study was obtained in both participating hospitals.

\section{RESULTS}

A total of 113 patients presented with an intussusception, 55 to the Juliana Children's Hospital and 58 to the Sophia Children's Hospital. There was a male dominance (3:2) and $84 \%$ were under 2 years of age. Presenting symptoms included vomiting (86\%), abdominal pain $(67 \%)$, and rectal blood loss (63\%). An abdominal mass was palpable in $35 \%$. The most commonly identified lead point appeared to be lymphoid hyperplasia, but in five cases a Meckel's diverticulum was responsible.

Peritonitis was present in 16. Of these bowel resection was necessary in seven, the other nine undergoing manual reduction. Of the seven requiring resection, six had presented with symptoms for more than 24 hours. However, of the nine in whom the intussusception could manually be reduced, six had symptoms for more then 24 hours.

Hydrostatic reduction was attempted in 97 (86\%), and was successful in $77(79 \%)$. There were 26/32 successful reductions with symptoms less than 12 hours, 30/37 with symptoms for between 12 and 24 hours, and 21/28 with symptoms for more than 24 hours (table 1). In the latter group $62 \%$ had symptoms for more than 48 hours, and the mean duration was 57 hours. In nine cases there was a recurrence of the intussusception within 12 hours of reduction. In four of these this was treated successfully by repeat hydrostatic reduction. The other five underwent laparotomy, and three required bowel resection (fig 1).

In 20 hydrostatic reduction was unsuccessful, including one case in which a bowel perforation occurred during the procedure. In 15 of these, resection of the intussusception was required; five of them had symptoms for more than 24 hours. In five of the 20, manual reduction was possible.

Table 1 Duration of symptoms and results of hydrostatic reduction

\begin{tabular}{lcccl}
\hline & $<\mathbf{1 2}$ hours & $\mathbf{1 2 - 2 4}$ hours & $>\mathbf{2 4}$ hours & Total \\
\hline Successful hydrostatic reduction & $26(81 \%)$ & $30(81 \%)$ & $21(75 \%)$ & 77 \\
Unsuccessful hydrostatic reduction & $6(19 \%)$ & $7(19 \%)$ & $7(25 \%)$ & 20 \\
Total & 32 & 37 & 28 & 97 \\
\hline
\end{tabular}




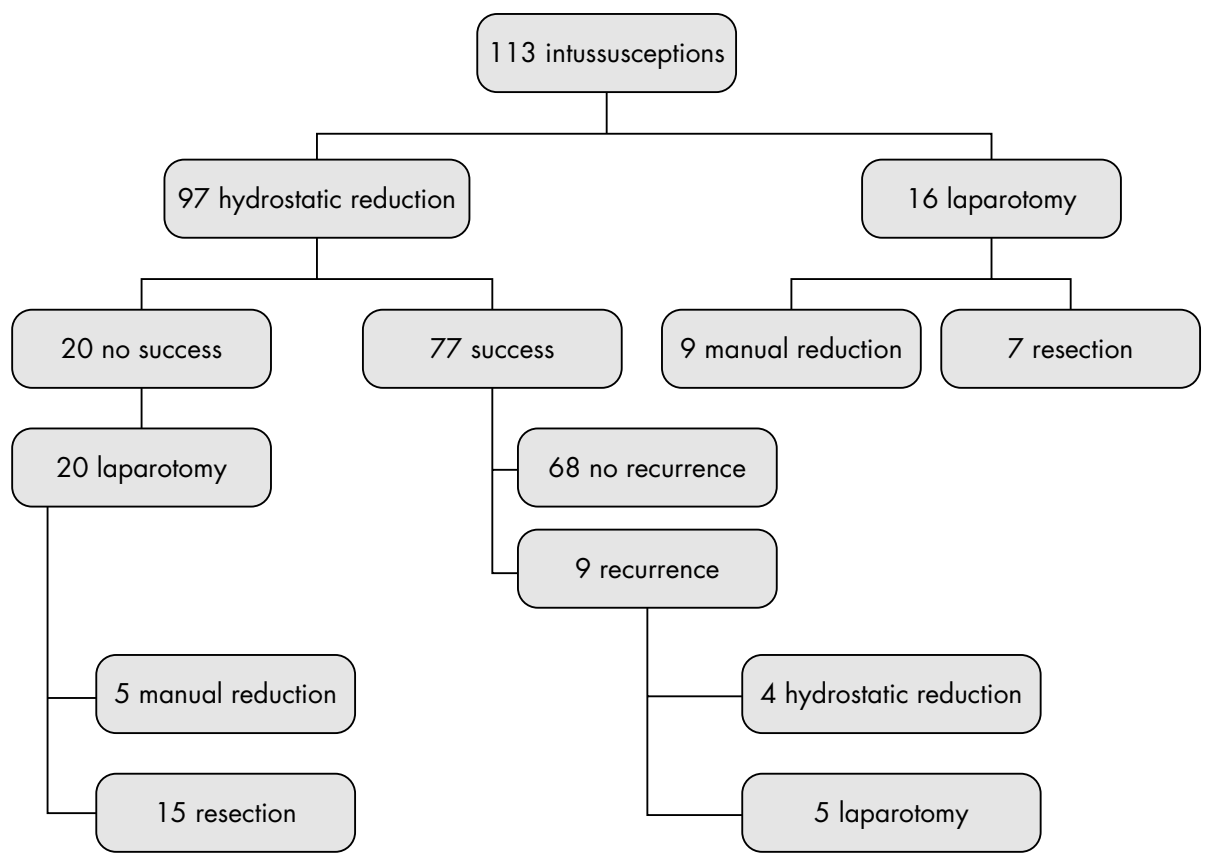

Figure 1 Summary of results.

\section{DISCUSSION}

The symptoms of intussusception are non-specific. ${ }^{4-6}$ In our population only $13 \%$ of the children presented with classical symptoms. Ultrasound is a reliable diagnostic tool with a sensitivity and a specificity close to $100 \%{ }^{7-11}$ The role of the abdominal radiograph is more controversial, ${ }^{12}{ }^{13}$ and its main value may be in excluding the presence of free air in the abdomen.

Non-operative reduction using barium or air contrast techniques is successful in about $75-90 \%$ of patients. ${ }^{11}$ In this study the success rate with hydrostatic reduction was $79 \%$. Several authors have reported that the success rate of hydrostatic reduction is lower and the risk of perforation risk higher in patients with symptoms for more than 48 hours, ${ }^{1-3}$ while others have reported that although the likelihood of hydrostatic reduction may be reduced, the risk of complication is no greater in patients with a longer duration of symptoms. ${ }^{14}$ In this study we did not find that the likelihood of successful hydrostatic reduction was less in those with more prolonged symptoms at presentation.

\section{Authors' affiliations}

E D van den Ende, J H Allema, P J Breslau, Red Cross and Juliana Children's Hospital, The Hague, Netherlands

F W J Hazebroek, Sophia Children's Hospital, Rotterdam, Netherlands

Competing interests: none declared

\section{REFERENCES}

1 West KW, Stephens B, Vane DW, et al. Intussusception: current management in infants and children. Surgery 1987;102:704-10.

2 Davis CF, Mc Gabe AJ, Raine PAM. The ins and outs of intussusception: history and management over the past fifty years. J Pediatr Surg 2003;38:60-4.

3 Reijnen JA, Festen C, van Roosmalen RP. Intussusception: factors related to treatment. Arch Dis Child 1990;65:871-3.

4 Daneman A, Navarro O. Intussusception. Part 1: A review of diagnostic approaches. Pediatr Radiol 2003;33:79-85.

5 Difiore JW. Intussusception. Semin Pediatric Surg 1999;8:214-20.

6 Yamamoto LG, Morita SY, Boychuk RB, et al. Stool appearance in intussusception: assessing the value of the term "currant jelly". Am J Emerg Med 1997; 15:293-8.

7 Hendrikson S, Blane CE, Koujok K, et al. The effect of screening sonography on the positive rate of enemas for intussusception. Pediatr Radiol 2003;33:190-3.

8 Daneman A, Alton DJ. Intussusception. Issues and controversies related to diagnosis and reduction. Radiol Clin North Am 1996;34:743-56.

9 Lim HK, Bae SH, Lee KH, et al. Assessment of reducibility of ileocolic intussusception in children: usefulness of color Doppler sonography. Radiology 1994;191:781-5.

10 Schmit P, Rohrschneider WK, Christmann D. Intestinal intussusception survey about diagnostic and nonsurgical therapeutic procedures. Pediatr Radiol 1999;29:752-61.

11 Navarro $O$, Dugougeat $F$, Kornecki $A$, et al. The impact of imaging in the management of intussusception owing to pathologic lead points in children. $A$ review of 43 cases. Pediatr Radiol 2000;30:594-603.

12 Ratcliff JF, Fong S, Cheong I, et al. Plain film diagnosis of intussusception: prevalence of the target sign. Am J Roentgenol 1992;158:619-21.

13 Sargent MA, Babyn P, Alton DJ. Plain abdominal radiography in suspected intussusception: a reassessment. Pediatr Radiol 1994;24:17-20.

14 Stephenson CA, Seibert JJ, Strain JD, et al. Intussusception: clinical and radiographic factors influencing reducibility. Pediatr Radiol 1989;20:57-60. 